\title{
Success of community-based directly observed TB treatment in Mongolia
}

Claudia C. Dobler ${ }^{1,2}$, Sarah Korver ${ }^{3}$, Ochirbat Batbayar ${ }^{3}$, Sodnomdarjaa Oyuntsetseg ${ }^{3}$, Bold Tsolmon $^{3}$, Cameron Wright ${ }^{4}$, Bekhbat Solongo $^{4}$, Ben J. Marais ${ }^{1,5}$

${ }^{1}$ NHMRC Centre of Research Excellence in Tuberculosis Control, University of Sydney, Australia; ${ }^{2}$ Department of Respiratory Medicine, Liverpool Hospital and South Western Sydney Clinical School, University of New South Wales, Sydney, Australia; ${ }^{3}$ Mongolian National Tuberculosis Programme, National Centre for Communicable Diseases, Ulaanbaatar, Mongolia; ${ }^{4}$ Mongolian Anti-Tuberculosis Association (MATA), Ulaanbaatar, Mongolia; ${ }^{5}$ Marie Bashir Institute for Infectious Diseases and Biosecurity (MBI), University of Sydney, Australia

Corresponding author:

Dr Claudia C. Dobler

Department of Respiratory Medicine, Liverpool Hospital, Elizabeth Street, Liverpool (Sydney), NSW 2034, Australia

Tel: (+61) 029828 4101. Fax: (+61) 029828 4102. e-mail cdobler@ med.usyd.edu.au

Running head: Community-DOT in Mongolia

Word count main text: 2495

Keywords: tuberculosis treatment, treatment completion, access to tuberculosis care 


\begin{abstract}
Background: Many countries restrict access to directly observed therapy (DOT) for tuberculosis through government health facilities. More innovative approaches are required to reduce non-adherence, improve patient outcomes and limit the risk of selecting drug-resistant strains.
\end{abstract}

Methods: We performed a retrospective cohort study in sputum smear-positive patients who were treated with community-DOT (either home-DOT or lunch-DOT) and conventional clinicDOT in Ulaanbaatar, the capital of Mongolia, in 2010-2011. We compared treatment success using community-based home-DOT versus conventional clinic-DOT and describe treatment completion rates using lunch-DOT, where DOT is provided with a free daily meal once sputum smear conversion has been documented.

Results: The overall treatment success among new sputum smear-positive TB patients was $85.1 \%(1,505 / 1,768)$. Patients receiving community-DOT had higher cure rates $(89.9 \%$, 294/327 versus $77.2 \%, 1,112 / 1441$, aOR 2.66 ; $95 \%$ CI 1.81 to 3.90) and higher treatment success $(93.6 \%, 306 / 327$ versus 83.2\%, 1,199/1,441; aOR 2.95; 95\% CI 1.85 to 4.71 , $\mathrm{p}<0.001)$ compared to clinic-DOT. Apart from 1 death, treatment completion was $100 \%$ among patients who received lunch-DOT after sputum smear conversion.

Conclusions: Community-DOT improved treatment success in Ulaanbaatar, Mongolia. It should be scaled up to be available for more patients and in all regions of the country. 


\section{INTRODUCTION}

Adherence to tuberculosis (TB) treatment is challenging because of the long duration of treatment- usually well beyond the point when patients feel better- and the number of drugs patients need to take. Non-adherence to TB treatment is known to be an important contributor to the selection of drug-resistant strains of Mycobacterium tuberculosis and subsequent treatment failure. ${ }^{1}$ Based on the need to ensure treatment adherence and limit the risk of ongoing TB transmission within communities, the World Health Organization (WHO) recommended directly observed therapy (DOT) in all patients treated for TB. ${ }^{2}$ DOT was a central part of the DOTS strategy and has become the standard of care in most countries.

National TB programmes (NTPs) usually restrict access to free TB treatment via specified government DOT centres, mostly local clinics. However, given sub-optimal TB treatment completion rates in many parts of the world, global TB control strategies require more innovative approaches for scaling up DOT and achieving stated targets. The value of community involvement to reduce barriers to care and ensuring TB treatment success is well recognized, ${ }^{3}$ but rarely operationalised.

Receiving DOT at the local clinic often requires a patient to give up part of their working day and neglect responsibilities at home. ${ }^{4-6}$ Patients might even have to choose between stable employment and TB treatment completion. ${ }^{4}$ These indirect opportunity costs, as well as direct travel costs and other charges can negatively influences adherence, even when TB treatment is provided free of charge. Strategies that reduce the total cost, direct and indirect, of TB treatment, such as decentralised community-based DOT, have the potential to improve treatment adherence. ${ }^{7}$ 
This study aimed to determine the effectiveness of community-based DOT compared to traditional clinic-DOT in Ulaanbaatar, the capital of Mongolia. We assessed the rates of treatment success with two community-based DOT strategies: home-DOT for the whole duration of treatment and a combination of initial clinic-DOT followed by lunch-DOT after patients had sputum smear conversion. With "home-DOT" community volunteers delivered TB treatment at home; with "lunch-DOT" community volunteers provided TB treatment after patients received a free meal.

\section{METHODS}

\section{Study setting}

Since Mongolia's transition to a market economy in the 1990s, following the collapse of socialism, the gap between rich and poor has widened continuously. While the mining boom in the mineral-rich nation has created a wealthy new elite, almost 30 percent of the population still live below the poverty line. ${ }^{8}$

Mongolia is one of the countries with the highest incidence of TB in the Western Pacific region with an incidence of drug-susceptible TB of 141/100,000 and an incidence of diagnosed multidrug-resistant TB of 7.5/100,000 (data from the Mongolian NTP, 2012). The number of notified TB cases (all forms) during the study period in Mongolia was 4,801 in 2010 and 4,533 in 2011 (data from the Mongolian NTP). A major strength of the Mongolian NTP is a dedicated network of approximately 300 volunteers, coordinated by the Mongolian Anti-Tuberculosis Association (MATA), who provide decentralised DOT. MATA conducts home-based and lunch-based DOT for around $25 \%$ of patients diagnosed with TB in Mongolia. 
The study was conducted in Ulaanbaatar, the capital of Mongolia and home to $45 \%$ of the Mongolian population. Eight districts of Ulaanbaatar that were served by the conventional clinic-based DOT programme as well as the community-based DOT programme were included in the study.

\section{Study design}

We extracted data on all sputum smear-positive TB patients aged $\geq 15$ years receiving DOT between 1 January 2010 and 31 December 2011 from eight districts in Ulaanbaatar from the Mongolian NTP TB register. Data analysed included age, gender, occupation, district of residence, treatment regimen, treatment category, duration of treatment and treatment outcome. Information on the allocated community-DOT strategy was obtained from MATA.

The two community based DOT strategies implemented by MATA included volunteers delivering TB medication to patients' homes every weekday (home-DOT) and poor and malnourished TB patients receiving a free meal at contracted cafeterias every weekday before taking their TB medication (lunch-DOT). Patients who received lunch-DOT were initially treated with conventional clinic DOT until documented sputum smear conversion. HomeDOT consisted of 5 days (Monday to Friday) of supervised treatment by a MATA volunteer and 2 days (Saturday and Sunday) of unsupervised treatment (patient self-administered) every week. Lunch-DOT consisted of 5 days (Monday to Friday) of supervised treatment with a free meal supervised by MATA volunteers at a cafe contracted by MATA and 2 days (Saturday and Sunday) of unsupervised treatment (patient self-administered) every week. Clinic-DOT consisted of 5 days (Monday to Friday) of supervised treatment at a TB clinic and 2 days (Saturday and Sunday) of unsupervised treatment (patient self-administered) 
every week.

Barring changes in circumstances of the patient or volunteer, for home DOT, the same volunteer was supervising a particular patient for the whole duration of treatment. For lunchDOT there was a permanent team of the same volunteers providing supervision of treatment at contracted cafes; for clinic-DOT there was a permanent team of the same health professionals (again barring change in the circumstances of clinic staff) providing supervision of treatment at a specific TB clinic.

To be eligible for home-DOT, patients must have lived at a permanent address in an area accessible to MATA volunteers; agreed to take their TB medication at a given time every weekday and presented a substantiated reason for being unable to attend dispensaries every weekday (e.g. poor health, transportation costs, familial or study/work commitments). Patients were eligible for lunch-DOT if they: 1) were able to come to a contracted cafeteria every weekday; 2) were socio-economically disadvantaged, defined as either being unemployed or having a disability, being from a family with two or more members diagnosed with TB, or being an elderly patient not having family caregivers, and; 3 ) were under- or malnourished as assessed by the treating doctor using their clinical judgment. Patients were only considered for lunch-DOT after sputum smear conversion. The type of DOT chosen was based on these criteria and following discussion between the treating doctor and patient. MATA staff were then contacted to arrange for patient follow up with home- or lunch-DOT.

All patients on community-DOT and clinic-DOT underwent a monthly clinical evaluation and sputum smear microscopy at the TB clinic. All patients received standard treatment regimens recommended by the WHO. 


\section{Community volunteers}

MATA volunteers comprised of lay persons trained in the basics of TB, and they were provided with a small monthly stipend; the Mongolian equivalent of around 30 US dollars. They were supervised by MATA staff and worked in close collaboration with TB clinic doctors and nurses who took responsibility for treatment decisions.

\section{Analysis}

Treatment outcomes were defined according to WHO guidelines. ${ }^{9}$ The primary outcome was treatment success defined as the sum of cured patients and patients who completed treatment. Only newly diagnosed sputum smear-positive patients (without prior history of TB treatment) were included in the comparative analysis to assess treatment success. Patients who were transferred in or out during the study period, i.e. they commenced or completed treatment outside the districts included in the study, were excluded from all analyses.

For the primary analysis, patients' outcome counted toward their originally assigned group (clinic-DOT or home-DOT) regardless of whether clinic-DOT patients crossed-over to lunchDOT after sputum smear conversion. This "intention to treat" analysis approach was chosen to avoid selection bias because only patients with sputum smear conversion (who were by definition highly likely to successfully complete treatment) were crossed-over to lunch-DOT.

Associations between treatment success and demographic and clinical characteristics were assessed as odds ratios (ORs) with $95 \%$ confidence intervals (95\% CIs) using logistic regression. The independent effect of potential predictors of treatment success was estimated using multivariate logistic regression. Multivariate analysis included adjustment for age, gender and occupation. Statistical analysis was performed using IBM SPSS Statistics 22 
(IBM, Armonk, NY, USA).

As data were collected as part of routine TB surveillance, this analysis was not considered research involving human subjects, and ethics approval was not required.

\section{RESULTS}

\section{Characteristics of the study cohort}

There were 2,181 sputum smear-positive patients including 1,714 (79\%) treated with clinicDOT only, $346(16 \%)$ treated with home-DOT and $121(5 \%)$ with initial clinic-DOT followed by lunch-DOT after sputum smear conversion. Newly diagnosed sputum smear-positive patients made up $81 \%(1,768 / 2,181)$ of the whole cohort with varying proportions among the treatment groups studied; $77 \%(1,324 / 1,714)$ among clinic-DOT, 95\% (327/346) among homeDOT and 97\% (117/121) among the lunch-DOT patients. The remaining patients received retreatment after the initial treatment had either been discontinued, had failed or the patient had disease recurrence. Table 1 summarises the demographic characteristics of the 1,768 newly diagnosed patients included in the analysis for treatment success.

Patients receiving lunch-DOT were older (median 36 years, range 15 to 72 years) than those treated with home-DOT (median 31 years, range 15 to 87) and clinic-DOT only (median 28 years, range 15 to 87 years). The combined unemployment rate among TB patients was high at $43.9 \%$. The proportion of unemployed people was highest in the lunch-DOT group $(61,52.1 \%)$ and lowest in the clinic-DOT group $(563,42.5 \%)$.

\section{Treatment outcomes in new sputum smear-positive patients}

The overall treatment success among all cases was $85.1 \%(1,505 / 1,768)$. Patients receiving 
home-DOT had higher cure rates $(89.9 \%, 294 / 327$ versus $77.2 \%, 1,112 / 1441)$ and treatment success $(93.6 \%, 306 / 327$ versus $83.2 \%, 1,199 / 1,441)$ than those on clinic-DOT. Apart from one death, treatment completion was $100 \%$ among patients who received lunch-DOT, after initial clinic-DOT. Table 2 summarizes treatment outcomes with different DOT delivery mechanisms.

The higher treatment success among patients in the home-DOT group compared to the clinicDOT group on univariate analysis (OR $2.94,95 \%$ CI 1.85 to $4.57, \mathrm{p}<0.001)$ was confirmed on multivariate analysis when adjusting for gender, age and occupation (aOR 2.95, 95\% CI 1.85 to $4.71, \mathrm{p}<0.001)$. Of all variables assessed, use of the home-DOT delivery mechanism had the greatest impact on treatment success. Multivariate analysis also confirmed higher cure rates among patients receiving home-DOT compared to patients receiving clinic-DOT (aOR 2.66; 95\% CI 1.81 to 3.90$)$.

Other factors significantly associated with higher rates of treatment success on uni- and multivariate analyses were female gender (aOR 1.63, 95\% CI 1.23 to 2.16 ), being employed (aOR $1.43,95 \%$ CI 1.07 to 1.92 ) and age less than 35 years (aOR 1.33, 95\% CI (1.01 to 1.77) (Table 3).

\section{DISCUSSION}

The key study finding is the significantly higher treatment success rates achieved with community-based DOT provided by volunteers in the patients' homes compared to traditional clinic-based DOT provided at a health facility. The potential value of community involvement in DOT provision has been recognised, but previous studies reported conflicting 
results.

A recent retrospective cohort study from Bangalore, India, showed higher treatment success among patients treated with community-DOT $(93 \%, 564 / 604)$ compared to clinic-DOT $(75 \%$, 951/1,260, RR $1.23,95 \%$ CI 1.19 to 1.28$).{ }^{10}$ However, treatment success rates were not adjusted for important confounders such as gender, age, occupation or socioeconomic status, and the fact that patients self-selected their preferred DOT delivery mechanism may have biased results. An earlier study from Brazil compared treatment success among patients living in a slum treated with community-DOT to patients living in other areas treated with clinic-DOT. ${ }^{11}$ Patients receiving community-DOT had higher treatment success than patients receiving clinic-DOT (OR 3.0, 95\% CI 1.9 to 4.8 versus OR $1.5,95 \%$ CI 0.9 to 2.4 ) after adjustment for potential confounders.

However, other studies that employed more rigorous study designs failed to demonstrate significant benefit of community-DOT compared to clinic-DOT. ${ }^{12-14}$ Two randomized controlled trials, both done in Tanzania, failed to find a significant difference in treatment success comparing community-DOT to clinic-DOT. ${ }^{15,16}$ In one of the trials, family members provided DOT, ${ }^{15}$ in the other trial DOT was given by trained community volunteers. The successful implementation of complex health care interventions such as the use of different DOT delivery mechanisms is dependent on local context, which explains why study results are likely to differ. In our study, the community volunteer programme was well embedded within Mongolian NTP activities and links with clinic staff were generally strong, providing the necessary medical backup - all of which we believe is essential for the success of a community-DOT programme. Further, community volunteers were supervised by salaried MATA staff on a weekly basis and received training in TB basics, which empowered them to 
share their knowledge about TB with patients.

The convenience to access treatment at home without the cost associated with travel to a central treatment facility and opportunity cost associated with this (e.g. the loss of income due to absence at work while attending the clinic) is likely a key factor in the success of community-DOT. The motivational support provided by a community-worker who develops a personal relationship with the patient over time is another possible contributor to increased treatment success. Further, community-workers usually come from the community they serve and are in a strong position to overcome socioeconomic and gender-specific barriers to completing TB treatment. However, this is likely to vary depending on the selection of community-workers, the training they receive and local social dynamics related to gender relationships and stigma etc.

Among poor and malnourished patients who received ongoing TB treatment together with a free meal, treatment completion was universal, apart from a single death that was not TB related. Patients in this particular risk group often experience poor treatment outcomes with traditional approaches, due to the costs associated with accessing treatment facilities, variable daily routines dependant on income generating opportunities and potential loss of income during clinic attendance. Additionally, malnutrition is a risk factor for TB mortality and delayed clearance of bacteria, making this group less likely to achieve treatment success. ${ }^{17}$ The evidence that incentives such as cash payments or free meals increase adherence to DOT is controversial. Some studies have demonstrated that food incentives improve adherence to drug treatment among socially marginalised patients with TB. ${ }^{18-20}$ A randomised controlled trial from Timor-Leste, however, did not find that the provision of food as a general incentive provided to all patients improved treatment outcomes. ${ }^{21}$ 
An important feature of the Mongolian lunch-DOT programme that may have contributed to treatment success was the involvement of a whole network of lunch providers at several decentralised locations, thus reducing barriers to accessing treatment. In the Mongolian lunch-DOT the TB drugs were given immediately after the patients had their meal. There is some evidence that ingestion of the TB medication with food may delay or moderately decrease the absorption of the drugs. Based on the wide therapeutic margin of the first line agents, however, this effect is of little clinical significance. ${ }^{22,23}$

Based on the fact that there was a significant difference in default rates between patients that commenced on clinic-DOT and switched to lunch-DOT after they achieved sputum clearance (0 patients defaulting) and those patients who remained on clinic-DOT for the whole duration of treatment ( $6.3 \%$ of patients defaulting), it seems likely that some defaults could have been avoided by using less stringent inclusion criteria for lunch-DOT and scaling up this community-based DOT modality to include more patients.

The overall treatment success of $83.2 \%$ of all patients commenced on clinic-DOT was below the international recommended goal of $85 \%$, and indicates a need to strengthen the conventional system to improve clinic-DOT treatment success in addition to scaling up community-DOT.

Our study was limited by its retrospective nature and potential for selection bias that can be avoided in a randomised controlled trial. To reduce the risk of selection bias, we only included new sputum smear-positive cases in the comparative analysis, since re-treatment patients were overrepresented in the clinic-DOT group. Further, we adjusted for important 
potential confounders such as gender, age and socioeconomic disadvantage (based on employment status). Importantly, none of the eligibility criteria for home-DOT introduced an obvious selection bias by for example excluding people who were perceived to be at risk of not completing treatment. The lunch-DOT strategy could not be directly compared to other DOT strategies because of inherent selection bias; only sputum smear converters were eligible for lunch-DOT. This was considered necessary to limit the risk of TB transmission, as patients could not wear face masks when eating. The excellent treatment outcomes achieved provides some confirmation that this approach can work in poor and malnourished patients, vulnerable to experience economic barriers to access centralised DOT.

There is increasing recognition that real-life data, such as the data obtained from our study, have certain advantages over data obtained from a more artificial trial setting. ${ }^{24}$ Decision makers request real life data on the effectiveness of health care interventions in a particular setting to better manage uncertainties associated with barriers to implementation and uptake of new health care interventions. The implementation of practice changes that require adaptation of health care delivery systems and infrastructure can be complex and relies on the collaboration of various stakeholders with different needs and perspectives. Careful analysis of real world programme implementation is important for decision makers to strategically prioritise new healthcare initiatives and funding.

\section{Conclusions}

Our study showed that home-DOT provided by dedicated community volunteers was associated with higher treatment success than traditional clinic-DOT, in a TB endemic setting with major service delivery challenges. Treatment completion rates among poor and malnourished patients who were offered a free meal with DOT at contracted cafes were 
virtually universal. Community-DOT should be scaled up to be available for more patients and in all regions of the country.

\section{Acknowledgements}

We thank all the staff of the Mongolian National TB programme and MATA for their dedication throughout this project. 


\section{References}

1. Weis SE, Slocum PC, Blais FX, et al. The effect of directly observed therapy on the rates of drug resistance and relapse in tuberculosis. N Engl J Med. 1994;330(17):1179-1184.

2. Frieden TR, Sbarbaro JA. Promoting adherence to treatment for tuberculosis: the importance of direct observation. Bull World Health Organ. 2007;85(5):407-409.

3. Maher D. The role of the community in the control of tuberculosis. Tuberculosis (Edinb). 2003;83(1):177-182.

4. Munro SA, Lewin SA, Smith HJ, Engel ME, Fretheim A, Volmink J. Patient adherence to tuberculosis treatment: a systematic review of qualitative research. PLoS medicine. 2007;4(7):e238.

5. Mauch V, Woods N, Kirubi B, Kipruto H, Sitienei J, Klinkenberg E. Assessing access barriers to tuberculosis care with the tool to Estimate Patients' Costs: pilot results from two districts in Kenya. BMC Public Health. 2011;11(1):43.

6. Needham DM, Godfrey-Faussett P. Economic barriers for tuberculosis patients in Zambia. The Lancet. 1996;348(9020):134-135.

7. Saunderson PR. An economic evaluation of alternative programme designs for tuberculosis control in rural Uganda. Soc Sci Med. 1995;40(9):1203-1212.

8. The World Bank. Mongolia - World Development Indicators. Available at: http://data.worldbank.org/country/mongolia Accessed 7 December2014.

9. World Health Organization: Definitions and reporting framework for tuberculosis - 2013 version. Geneva, Switzerland: World Health Organization; 2013.

10. Tripathy S, Kumar P, Sagili K, Enarson D. Effectiveness of a community-based observation of anti-tuberculosis treatment in Bangalore City, India, 2010-2011. Public Health Action. 2013;3(3):230-234.

11. Cavalcante S, Soares E, Pacheco A, Chaisson R, Durovni B, Team DE. Community DOT for tuberculosis in a Brazilian favela: comparison with a clinic model. The International Journal of Tuberculosis and Lung Disease. 2007;11(5):544-549.

12. Kironde S, Meintjies M. Tuberculosis treatment delivery in high burden settings: does patient choice of supervision matter? The International Journal of Tuberculosis and Lung Disease. 2002;6(7):599-608.

13. Singh AA, Parasher D, Shekhavat GS, Sahu S, Wares DF, Granich R. Effectiveness of urban community volunteers in directly observed treatment of tuberculosis patients: a field report from Haryana, North India [Notes from the Field]. The International Journal of Tuberculosis and Lung Disease. 2004;8(6):800-802.

14. Kamolratanakul P, Sawert H, Lertmaharit S, et al. Randomized controlled trial of directly observed treatment (DOT) for patients with pulmonary tuberculosis in Thailand. Transactions of the Royal Society of Tropical Medicine and Hygiene. 1999;93(5):552557.

15. Wandwalo E, Kapalata N, Egwaga S, Morkve O. Effectiveness of community-based directly observed treatment for tuberculosis in an urban setting in Tanzania: a randomised controlled trial. The International Journal of Tuberculosis and Lung Disease. 2004;8(10):1248-1254.

16. Lwilla F, Schellenberg D, Masanja H, et al. Evaluation of efficacy of community-based vs. institutional-based direct observed short-course treatment for the control of tuberculosis in Kilombero district, Tanzania. Trop Med Int Health. 2003;8(3):204-210.

17. Gupta KB, Gupta R, Atreja A, Verma M, Vishvkarma S. Tuberculosis and nutrition. Lung India: official organ of Indian Chest Society. 2009;26(1):9. 
18. Gärden B, Samarina A, Stavchanskaya I, et al. Food incentives improve adherence to tuberculosis drug treatment among homeless patients in Russia. Scandinavian journal of caring sciences. 2013;27(1):117-122.

19. Bock N, Sales R, Rogers T, DeVoe B. A spoonful of sugar...: improving adherence to tuberculosis treatment using financial incentives [Notes from the Field. The International Journal of Tuberculosis and Lung Disease. 2001;5(1):96-98.

20. Cantalice Filho JP. Food baskets given to tuberculosis patients at a primary health care clinic in the city of Duque de Caxias, Brazil: effect on treatment outcomes. Jornal Brasileiro de Pneumologia. 2009;35(10):992-997.

21. Martins N, Morris P, Kelly PM. Food incentives to improve completion of tuberculosis treatment: randomised controlled trial in Dili, Timor-Leste. Bmj. 2009;339.

22. World Health Organization. Guidelines for treatment of tuberculosis. 2010. Available at http://www.who.int/tb/publications/2010/9789241547833/en/ Acessed 7 December 2014.

23. Treatment of tuberculosis. MMWR Recomm Rep. 2003;52(RR-11):1-77.

24. Annemans L, Aristides M, Kubin M. Real-life data: A growing need. ISPOR connections. 2007. 
Table 1 Characteristics of new sputum smear-positive cases stratified by DOT delivery mechanism

\begin{tabular}{lcccc}
\hline \multicolumn{1}{c}{ Characteristics } & All & $\begin{array}{c}\text { clinic-DOT } \\
\text { (without } \\
\text { lunch-DOT } \\
\text { patients) }\end{array}$ & home-DOT & lunch-DOT \\
& $\mathrm{n}(\%)$ & $\mathrm{n}(\%)$ & $\mathrm{n}(\%)$ & $\mathrm{n}(\%)$ \\
\hline Total & $\mathbf{1 , 7 6 8 ( 1 0 0 )}$ & $\mathbf{1 , 3 2 4 ( \mathbf { 7 4 . 9 } )}$ & $\mathbf{3 2 7}(\mathbf{1 8 . 5})$ & $\mathbf{1 1 7}(\mathbf{6 . 6})$ \\
Gender & $974(55.1)$ & $744(56.2)$ & $167(51.1)$ & $63(53.8)$ \\
Male & $794(44.9)$ & $580(43.8)$ & $160(48.9)$ & $54(46.2)$ \\
Female & & & & \\
Age in years & $1,053(59.6)$ & $797(60.2)$ & $202(61.8)$ & $54(46.2)$ \\
15 to 34 & $562(31.8)$ & $421(31.8)$ & $92(28.1)$ & $49(41.9)$ \\
35 to 54 & $153(8.7)$ & $106(8.0)$ & $33(10.1)$ & $14(12.0)$ \\
$\geq 55$ & & & & \\
Occupation & $486(27.5)$ & $394(29.8)$ & $76(23.2)$ & $16(13.7)$ \\
Employed & & & & \\
(incl. self-employed) & & & & \\
Unemployed & $777(43.9)$ & $563(42.5)$ & $153(46.8)$ & $61(52.1)$ \\
Retired & $124(7.0)$ & $86(6.5)$ & $30(9.2)$ & $8(6.8)$ \\
Student & $285(16.1)$ & $208(15.7)$ & $54(16.5)$ & $23(19.7)$ \\
$\begin{array}{l}\text { Disability pension } \\
\text { Unknown }\end{array}$ & $70(4.0)$ & $49(3.7)$ & $12(3.7)$ & $9(7.7)$ \\
\hline
\end{tabular}


Table 2 Treatment outcomes according to DOT delivery mechanism

\begin{tabular}{|c|c|c|c|c|c|c|c|}
\hline $\begin{array}{l}\text { DOT } \\
\text { regimen }\end{array}$ & Total & Cured (\%) & $\begin{array}{c}\text { Completed } \\
(\%)\end{array}$ & $\begin{array}{l}\text { Treatment } \\
\text { success } \\
\text { (cured and } \\
\text { completed } \\
(\%)\end{array}$ & $\begin{array}{c}\text { Died } \\
(\%)\end{array}$ & $\begin{array}{c}\text { Failure } \\
(\%)\end{array}$ & $\begin{array}{c}\text { Defaulted } \\
(\%)\end{array}$ \\
\hline All & 1,768 & $1,406(79.5)$ & $99(5.6)$ & $1,505(85.1)$ & $29(1.6)$ & $140(7.9)$ & $94(5.3)$ \\
\hline Clinic-DOT* & 1,441 & $1,112(77.2)$ & $87(6.0)$ & $1,199(83.2)$ & $24(1.7)$ & $127(8.8)$ & $91(6.3)$ \\
\hline Home-DOT & 327 & 294 (89.9) & $12(3.7)$ & 306 (93.6) & $5(1.5)$ & $13(4.0)$ & $3(0.9)$ \\
\hline Lunch-DOT $^{\dagger}$ & 117 & $112(95.7)$ & $4(3.4)$ & $116(99.1)$ & $1(0.9)$ & 0 & 0 \\
\hline
\end{tabular}

* including patients who crossed-over to lunch-DOT after sputum smear conversion

$\dagger$ Patients in the lunch-DOT group were commenced on clinic-DOT, followed by lunch-DOT after smear conversion 
Table 3 Uni- and multivariate analysis of factors associated with treatment success

\begin{tabular}{|c|c|c|c|c|c|}
\hline Characteristic & $\begin{array}{l}\text { Treatment } \\
\text { success }\end{array}$ & $\begin{array}{c}\text { unadjusted } \\
\text { odds ratio } \\
(95 \% \mathrm{CI})\end{array}$ & $P$ value & $\begin{array}{l}\text { adjusted odds } \\
\text { ratio }(95 \% \mathrm{CI})\end{array}$ & $P$ value \\
\hline \multicolumn{6}{|l|}{$\begin{array}{l}\text { DOT delivery } \\
\text { mechanism }\end{array}$} \\
\hline $\begin{array}{l}\text { Clinic-DOT } \\
\text { (including lunch- } \\
\text { DOT patients) }\end{array}$ & $\begin{array}{c}1,199 / 1,441 \\
(83.2 \%)\end{array}$ & 1.00 (Ref) & & 1.00 (Ref) & \\
\hline Home-DOT & $\begin{array}{l}306 / 327 \\
(93.6 \%)\end{array}$ & $\begin{array}{c}2.94 \\
(1.85 \text { to } 4.67)\end{array}$ & $<0.001$ & $\begin{array}{c}2.95 \\
(1.85 \text { to } 4.71)\end{array}$ & $<0.001$ \\
\hline \multicolumn{6}{|l|}{ Gender } \\
\hline Male & $\begin{array}{l}799 / 974 \\
(82.0 \%)\end{array}$ & 1.00 (Ref) & & 1.00 (Ref) & \\
\hline Female & $\begin{array}{l}706 / 794 \\
(88.9 \%)\end{array}$ & $\begin{array}{c}1.76 \\
(1.33 \text { to } 2.31)\end{array}$ & $<0.001$ & $\begin{array}{c}1.64 \\
(1.24 \text { to } 2.18)\end{array}$ & 0.001 \\
\hline \multicolumn{6}{|l|}{ Age (years) } \\
\hline$\geq 35$ & $\begin{array}{l}585 / 715 \\
(81.8 \%)\end{array}$ & 1.00 (Ref) & & 1.00 (Ref) & \\
\hline$<35$ & $\begin{array}{c}920 / 1053 \\
(87.4 \%)\end{array}$ & $\begin{array}{c}1.54 \\
(1.18 \text { to } 2.00)\end{array}$ & 0.001 & $\begin{array}{c}1.33 \\
(1.01 \text { to } 1.77)\end{array}$ & 0.046 \\
\hline \multicolumn{6}{|l|}{ Employment } \\
\hline Unemployed & $\begin{array}{l}803 / 971 \\
(82.7 \%)\end{array}$ & 1.00 (Ref) & & 1.00 (Ref) & \\
\hline Employed, student & $\begin{array}{l}679 / 771 \\
(88.1 \%)\end{array}$ & $\begin{array}{c}1.54 \\
(1.17 \text { to } 2.03)\end{array}$ & 0.002 & $\begin{array}{c}1.46 \\
(1.09 \text { to } 1.95)\end{array}$ & 0.011 \\
\hline
\end{tabular}

